\title{
Salidroside protects against hydrogen peroxide-induced injury in HUVECs via the regulation of REDD1 and mTOR activation
}

\author{
MAO-CHUN XU ${ }^{1}$, HAI-MING SHI ${ }^{1}$, HAO WANG $^{2}$ and XIU-FANG GAO ${ }^{1}$ \\ ${ }^{1}$ Department of Cardiology, Huashan Hospital of Fudan University, Shanghai 200040; \\ ${ }^{2}$ The Key Laboratory of Molecular Medicine, Ministry of Education, Shanghai Medical School of Fudan University, \\ Shanghai 200032, P.R. China
}

Received January 13, 2013; Accepted April 25, 2013

DOI: $10.3892 / \mathrm{mmr} .2013 .1468$

\begin{abstract}
Antioxidative therapy is considered an effective strategy for treating oxidative stress-induced apoptosis in cardiovascular diseases. Salidroside has been used as an antioxidative therapy for oxidative injury in cardiac diseases. However, the mechanism underlying its antioxidant effect is poorly understood. The present study aimed to investigate the pharmacological effects of salidroside on cultured human umbilical vein endothelial cells (HUVECs) under conditions of oxidative injury induced by hydrogen peroxide $\left(\mathrm{H}_{2} \mathrm{O}_{2}\right)$ and the underlying mechanisms in vitro. HUVECs pretreated with or without salidroside for $24 \mathrm{~h}$ were exposed to $\mathrm{H}_{2} \mathrm{O}_{2}$-induced oxidative stress conditions for $6 \mathrm{~h}$ and then cell viability, apoptosis, HIF-1 $\alpha$, regulated in development and DNA damage responses-1 (REDD1) and the PI3K/Akt/mTOR pathway were investigated. The results demonstrated that salidroside effectively attenuated $\mathrm{H}_{2} \mathrm{O}_{2}$-impaired cell viability and the production of reactive oxygen species (ROS) in a concentration-dependent manner. Reduced $\mathrm{H}_{2} \mathrm{O}_{2}$-induced apoptosis and activation of the cellular PI3K/Akt/mTOR pathway were demonstrated in HUVECs pretreated with salidroside. Furthermore, the level of REDD1, a direct regulator of mitochondrial metabolism, significantly increased in parallel with the level of HIF-1 $\alpha$ following pretreatment with salidroside. The antioxidative effect of salidroside was abrogated in REDD1 knockdown cells. However, LY294002, a PI3K inhibitor, attenuated the anti-apoptotic effect of salidroside and blocked the increase of Akt and mTOR; however, did not affect the antioxidative effect of salidroside. These findings suggested that salidroside was capable of protecting HUVECs against $\mathrm{H}_{2} \mathrm{O}_{2}$-induced
\end{abstract}

Correspondence to: Dr Xiu-Fang Gao, Department of Cardiology, Huashan Hospital of Fudan University, 12 Urumuqi Road, Shanghai 200040, P.R. China

E-mail: sophiakao@126.com

Key words: salidroside, oxidative injury, REDD1, mTOR, apoptosis, signal transduction apoptosis by activating the PI3K/Akt/mTOR-dependent pathway and inhibiting ROS production by activating REDD1.

\section{Introduction}

There is evidence that oxidative stress is a major stimulus in the pathogenesis of cardiovascular diseases, including atherosclerosis, hypertension, myocardial infarction and heart failure $(1,2)$. Hypoxia and ischemia may increase the production of intracellular reactive oxygen species (ROS) and consequently induce cardiomyocyte necrosis and apoptosis $(3,4)$. Therefore, the development of an effective antioxidant strategy to reduce oxidative stress, which has attracted considerable interest from investigators, may provide insights into delaying or preventing cardiomyocyte death in ischemic heart diseases (5).

The mammalian target of rapamycin (mTOR) is the central controlling mechanism of cellular growth and metabolism. mTOR stimulates protein synthesis and cell growth through the inhibition of eukaryotic initiation factor $4 \mathrm{E}-$ binding protein-1 (4EBP1) and the activation of ribosomal protein S6 kinase (S6K) (6-8). The Akt-mediated activation of the mTOR pathway is a major regulator of the cellular response to hypoxia and other microenvironmental stresses (9). Several proteins are involved in the regulation of mTORC1 activity, including proline-rich Akt substrate of $40 \mathrm{kDa}$ (PRAS40), DEP domain-containing mTOR-interacting protein (DEPTOR) or regulated in development and DNA damage responses-1 (REDD1) (10-12).

REDD1, as a hypoxia-regulated HIF-1 target gene, is important in the TSC1/TSC2-mediated inhibition of mTOR (13). REDD1 is markedly induced by numerous stress stimuli, including hypoxia, oxidative stress and energy depletion. More recent studies have suggested that REDD1 functions as a direct regulator of mitochondrial metabolism; however, the molecular mechanisms by which cell stress and hypoxia induce REDD1 expression have yet to be determined $(14,15)$.

Salidroside (Fig. 1), as an adaptogen purified from Rhodiola rosea ( $R$. rosea), has been reported to exert antioxidative, anti-aging, neuroprotective and cardioprotective effects, implying that salidroside may play a central role in the alleviation of mitochondrial-generated ROS and modulation of mitochondrial-related apoptosis signaling in multiple 
types of cells (16). A previous study has demonstrated that pretreatment with salidroside upregulated the HIF-1 $\alpha$ protein and induced its translocation in order to protect cardiomyocytes against hypoxia-induced injury (17). Nevertheless, the mechanism underlying its antioxidative effects remains unclear and requires further investigation.

In the present study, we investigated the potential antiapoptosis activity of salidroside using human umbilical vein endothelial cells (HUVECs). Our results indicated that salidroside markedly inhibits hydrogen peroxide $\left(\mathrm{H}_{2} \mathrm{O}_{2}\right)$-induced HUVEC apoptosis and ROS production. Furthermore, we demonstrated that the anti-apoptosis effect of salidroside may be mediated by activation of the PI3K/Akt/mTOR pathway. Furthermore, the antioxidative effect of salidroside is dependent on REDD1 activity and requires the transcription factor HIF-1 $\alpha$.

In conclusion, our results demonstrated that the activation of REDD1 by salidroside affects cell viability and ROS production, and may be a promising candidate for antioxidant therapy in cardiovascular disease.

\section{Materials and methods}

Materials. Salidroside, an active component of the medicinal plant $R$. rosea, with $99 \%$ purity confirmed by HPLC, was purchased from the National Institute for the Control of Pharmaceutical and Biological Products (Beijing, China); 2',7'-dichlorodihydrofluorescein diacetate $\left(\mathrm{H}_{2} \mathrm{DCFDA}\right)$ and Lipofectamine ${ }^{\mathrm{TM}} 2000$ were purchased from Invitrogen Life Technologies (Carlsbad, CA, USA); FITC-conjugated annexin-V was purchased from BD Biosciences (San Jose, CA, USA); and LY294002 was purchased from Sigma-Aldrich (St. Louis, MO, USA).

Cell culture and treatment. HUVECs (ATCC, Teddington, UK) were cultured in F-12K medium with $100 \mathrm{U} / \mathrm{ml}$ penicillin, $100 \mu \mathrm{g} / \mathrm{ml}$ streptomycin, $2 \mathrm{mM}$ L-glutamine, $30 \mu \mathrm{g} / \mathrm{ml}$ endothelial cell growth supplement and $10 \% \mathrm{FBS}$ at $37^{\circ} \mathrm{C}$ in $5 \% \mathrm{CO}_{2}$ and $95 \%$ humidity. For $\mathrm{H}_{2} \mathrm{O}_{2}$ treatment, cells were first screened with increasing doses of $\mathrm{H}_{2} \mathrm{O}_{2}$ and the optimal concentration $(200 \mu \mathrm{mol})$ was selected for further experiments. For antioxidant treatment, the optimal concentration $(100 \mu \mathrm{g} / \mathrm{ml})$ of salidroside was selected for further experiments. Cells were cultured with salidroside or a saline vehicle for $24 \mathrm{~h}$, followed by $\mathrm{H}_{2} \mathrm{O}_{2}(200 \mu \mathrm{mol})$ treatment. For LY294002 treatment, cells were cultured with salidroside followed by LY294002 and $\mathrm{H}_{2} \mathrm{O}_{2}$ treatment.

Plasmid construction, lentiviral packaging and infection. To silence REDD1 expression, we constructed a vector-based REDD1-shRNA to interfere with the expression of REDD1. The oligonucleotides used were 5'-CCGGTGATGCCTAGCC AGTTGGTAACTCGAGTTACCAACTGGCTAGGCATCAT TTTTG-3' and 5'-AATTCAAAAATGATGCCTAGCCAGTT GGTAACTCGAGTTACCAACTGGCTAGGCATCA-3', and were cloned into the AgeI and EcoRI sites of the pLKO.1-puro vector (Sigma Aldrich). Lentiviruses were created by co-transfecting HEK 293 T cells with a packaging vector. Lentiviral supernatants were collected $48 \mathrm{~h}$ post-transfection and target cells were infected with $3 \mathrm{ml}$ virus supernatant containing $8 \mu \mathrm{g} / \mathrm{ml}$ polybrene for $24 \mathrm{~h}$. The media was

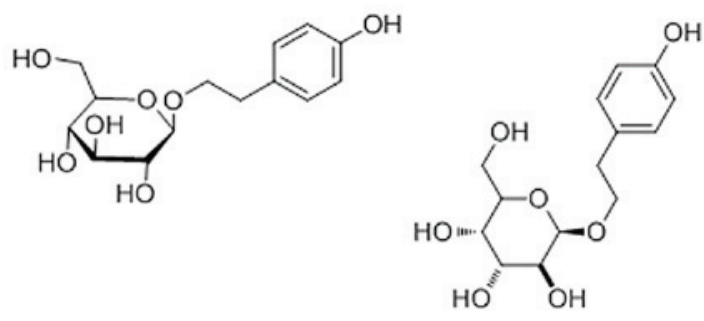

Figure 1. Chemical structure of salidroside (p-hydroxyphenethyl- $\beta$ D-glucoside).

replaced with complete medium. Puromycin $(3 \mu \mathrm{g} / \mathrm{ml})$ was added to the cell culture media two days after infection. The knockdown efficiency was monitored by western blot analysis. The formation of REDD1-shRNA was confirmed using DNA sequencing.

Cell viability assay. Cells were seeded in 96-well culture plates at $10^{4}$ cells/well and allowed to attach to the plates. The culture medium was removed and renewed with fresh medium containing $\mathrm{H}_{2} \mathrm{O}_{2}$ at various concentrations, ranging from $0-400 \mu \mathrm{M}$ and incubated for $6 \mathrm{~h}$. For the salidroside assays, cells were pretreated with salidroside for $24 \mathrm{~h}$ prior to the addition of $\mathrm{H}_{2} \mathrm{O}_{2}$. Cells receiving saline served as a vehicle control and were equivalent to no treatment. The Cell Counting kit-8 (CCK-8) reagent (Sigma Aldrich) was added and incubated at $37^{\circ} \mathrm{C}$ for $2 \mathrm{~h}$ according to the manufacturer's instructions. Optical density (OD) values at $450 \mathrm{~nm}$ were read by a microplate reader. Each experiment was performed three times independently.

Flow cytometric assays. To evaluate apoptosis in HUVECs, flow cytometric analysis was performed using annexin-V. Cells were treated with $100 \mu \mathrm{g} / \mathrm{ml}$ salidroside or saline for $24 \mathrm{~h}$, then $200 \mu \mathrm{mol} \mathrm{H}_{2} \mathrm{O}_{2}$ was added. Cells were detached by exposure to trypsin $6 \mathrm{~h}$ later. Apoptosis was detected in cells washed with PBS by staining with annexin-V and propidium iodide according to the manufacturer's instructions (BD Biosciences). Stained cells were analyzed using flow cytometry with CellQuest (Becton-Dickinson, Franklin Lakes, NJ, USA) software.

The cells, which had been treated or remained untreated with salidroside or $\mathrm{H}_{2} \mathrm{O}_{2}$, were incubated with a chloromethyl derivative of $\mathrm{H}_{2}$ DCFDA (CM- $\mathrm{H}_{2}$ DCFDA; Invitrogen Life Technologies) in the dark for $30 \mathrm{~min}$ at $37^{\circ} \mathrm{C}$. Following washing, the ROS level of cells was immediately analyzed by flow cytometry.

Protein extraction andwestern blot analysis. Cells were washed three times with ice-cold PBS and subsequently lysed in RIPA buffer containing $1 \mathrm{mM}$ PMSF. The supernatant was collected and the protein concentration of each sample was determined using a BCA Protein Assay kit (Pierce Biotechnology, Inc., Rockford,IL,USA) according to the manufacturer's instructions. Total proteins $(50 \mu \mathrm{g})$ were loaded onto $10 \%$ sodium dodecyl sulfate-polyacrylamide gels for electrophoresis and transferred onto 0.22- $\mu \mathrm{m}$ PVDF membranes (Millipore, Billerica, MA, USA), which were blocked with $8 \%$ non-fat milk. The primary antibodies used to probe the membranes included 
anti-HIF-1 $\alpha$ [Cell Signaling Technology, Inc. (CST), Beverley, MA, USA; \#3716], anti-REDD1 (ProteinTech Group, Chicago IL, USA; 10638-1-AP), anti-Akt (CST, \#9272), anti-p-Akt (CST, \#9271), anti-p-p70S6K (CST, \#6198), anti-p-mTOR (CST, \#2971) and anti-GAPDH (ProteinTech Group, 60004-1-Ig) at $4^{\circ} \mathrm{C}$ overnight. Following washing, the membranes were incubated for $1 \mathrm{~h}$ at room temperature with horseradish peroxidase-conjugated secondary antibody. Proteins were detected using an advanced enhanced chemiluminescence (ECL) system (GE Healthcare, Chalfont St. Giles, UK).

Statistical analysis. The results of all experiments are expressed as the means \pm SD from individual experiments. Data were compared using the Student's t-test using GraphPad Prism 5.0 software (GraphPad Software Inc., San Diego, CA, USA). $\mathrm{P}<0.05$ was considered to indicate a statistically significant difference.

\section{Results}

Salidroside attenuates $\mathrm{H}_{2} \mathrm{O}_{2}$-induced HUVEC cytotoxicity and excessive generation of ROS. In order to investigate the effect of salidroside on the growth of endothelial cells, we evaluated the effect of salidroside on the viability of HUVECs using the CCK-8 assay. $\mathrm{H}_{2} \mathrm{O}_{2}(50-400 \mu \mathrm{mol})$ significantly decreased the cell viability of HUVECs in a concentration-dependent manner ( $\mathrm{P}<0.01$; Fig. 2A). When exposed to $200 \mu \mathrm{mol} \mathrm{H}_{2} \mathrm{O}_{2}$ for $6 \mathrm{~h}$, HUVEC viability $(61.9 \pm 4.1 \%, \mathrm{n}=3)$ decreased by $38.1 \%$ compared with the control group $(\mathrm{P}<0.01$, both $\mathrm{n}=3)$. Therefore, $200 \mu \mathrm{mol}$ of $\mathrm{H}_{2} \mathrm{O}_{2}$ was used as the optimal concentration in the subsequent experiments. To examine the concentration-dependent effect of salidroside, cells were pretreated with 20,50 or $100 \mu \mathrm{g} / \mathrm{ml}$ salidroside or saline vehicle for $24 \mathrm{~h}$ prior to being exposed to $200 \mu \mathrm{mol} \mathrm{H}_{2} \mathrm{O}_{2}$ for $6 \mathrm{~h}$. The decrease in HUVEC viability induced by $\mathrm{H}_{2} \mathrm{O}_{2}$ was improved significantly by treatment with salidroside in a concentration-dependent manner. A significant increase in cell viability was evident following pretreatment with $100 \mu \mathrm{g} / \mathrm{ml}$ of salidroside (Fig. 2B). The optimal concentration $(100 \mu \mathrm{g} / \mathrm{ml})$ of salidroside was selected for further experiments. In addition, salidroside at each concentration applied alone did not cause any apparent cytotoxicity (data not shown). The dichlorofluorescein (DCFH) assay results indicated that salidroside reduced the levels of ROS in HUVECs under $\mathrm{H}_{2} \mathrm{O}_{2}$. When HUVECs were subjected to $\mathrm{H}_{2} \mathrm{O}_{2}$ for $6 \mathrm{~h}$, the concentration of intracellular ROS increased, compared with the control group $(\mathrm{P}<0.01$; Fig. $2 \mathrm{C})$. However, preincubation with $100 \mu \mathrm{g} / \mathrm{ml}$ salidroside for $24 \mathrm{~h}$ followed by coincubation with $200 \mu \mathrm{mol} \mathrm{H}_{2} \mathrm{O}_{2}$ for a further $6 \mathrm{~h}$ markedly decreased the $\mathrm{H}_{2} \mathrm{O}_{2}$-induced ROS level. ROS production decreased by $22.9 \%$ compared with cells treated with $\mathrm{H}_{2} \mathrm{O}_{2}$ alone $(\mathrm{P}<0.01$; Fig. 2D).

Salidroside inhibits $\mathrm{H}_{2} \mathrm{O}_{2}$-induced HUVEC apoptosis. CCK-8 does not discriminate between necrosis and apoptosis. We evaluated the possible anti-apoptotic effect of salidroside on HUVECs subjected to $\mathrm{H}_{2} \mathrm{O}_{2}$ using annexin-V/PI double staining and flow cytometry analysis. In the control group, the majority of cells were viable $(93.7 \%) . \mathrm{H}_{2} \mathrm{O}_{2}$ increased the percentage of apoptotic cells. Compared with the control group, $6 \mathrm{~h}$ stimulation of $\mathrm{H}_{2} \mathrm{O}_{2}$-induced apoptosis produced a
A

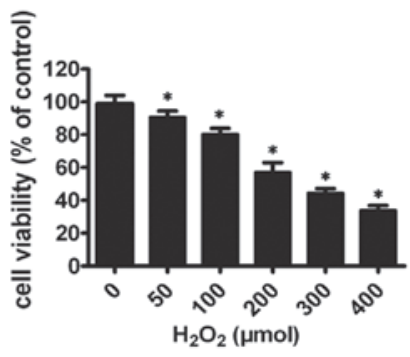

B

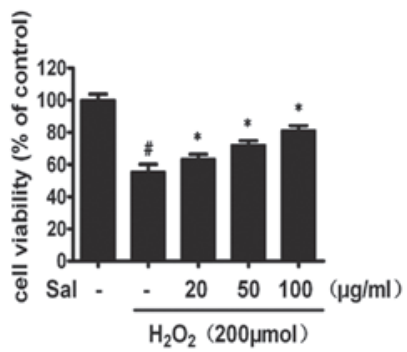

C

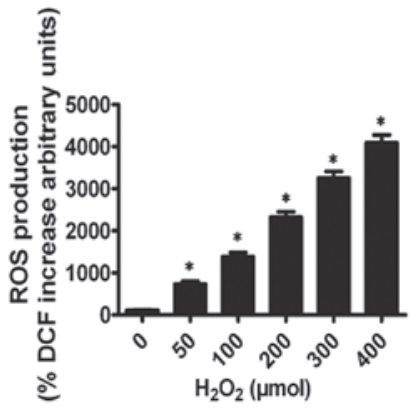

D

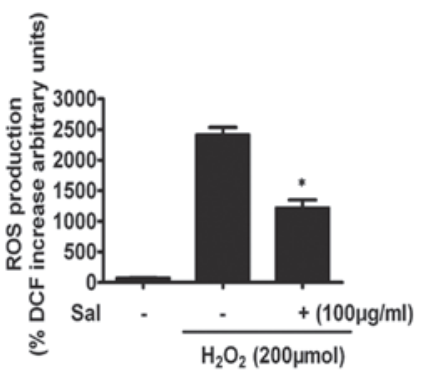

Figure 2. Sal attenuated $\mathrm{H}_{2} \mathrm{O}_{2}$-induced $\mathrm{HUVEC}$ cytotoxicity and excessive generation of ROS. (A) Concentration-dependent toxic effect of $\mathrm{H}_{2} \mathrm{O}_{2}$ on the viability of HUVECs. (B) Effect of Sal on $\mathrm{H}_{2} \mathrm{O}_{2}$-induced HUVEC death (C) ROS contents in HUVECs under $\mathrm{H}_{2} \mathrm{O}_{2}$ stimulation. (D) Changes in ROS induced by $\mathrm{H}_{2} \mathrm{O}_{2}$ alone or in combination in the absence or presence of Sal, respectively. All data are presented as the means \pm SEM of the three experiments and each included triplicate sets. ${ }^{*} \mathrm{P}<0.01$ vs. control group; ${ }^{~} \mathrm{P}<0.01$ vs. $100 \mu \mathrm{g} / \mathrm{ml}$ Sal group. Sal, salidroside; HUVECs, human umbilical vein endothelial cells; ROS, reactive oxygen species.

5.3-fold increase in apoptotic cells $(6.5 \pm 0.8 \%, \mathrm{P}<0.01, \mathrm{n}=3$; Fig. 3A and B). Pretreatment with salidroside for $24 \mathrm{~h}$ and cotreatment with $\mathrm{H}_{2} \mathrm{O}_{2}$ for a further $6 \mathrm{~h}$ significantly attenuated the apoptosis induced by $\mathrm{H}_{2} \mathrm{O}_{2}$, with $51.5 \%$ fewer apoptotic cells compared with the cells treated with $\mathrm{H}_{2} \mathrm{O}_{2}$ alone $(\mathrm{P}<0.01$, $\mathrm{n}=4$; Fig. 3C and D).

Salidroside increases the expression of REDD1 protein and activates the Akt/mTOR pathway. HIF-1 $\alpha$ reduces the production of ROS under hypoxic conditions, which mediate the cells adaptive response to hypoxia and ischemic. It is doubtful as to whether REDD1 is also associated with the process of 
A

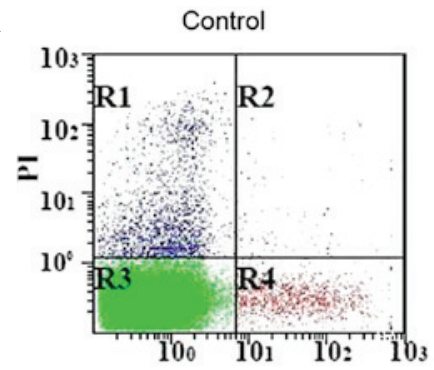

Annexin V-FITC

B

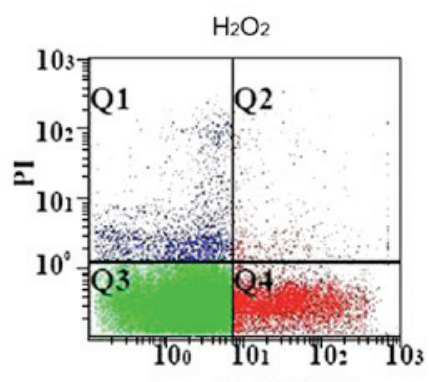

Annexin V-FITC

C

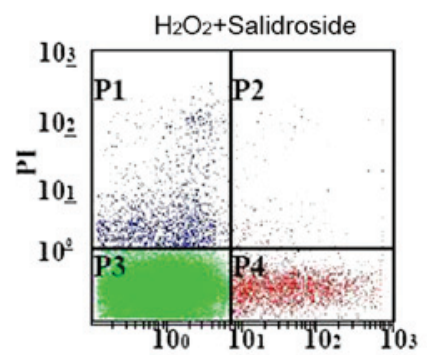

D

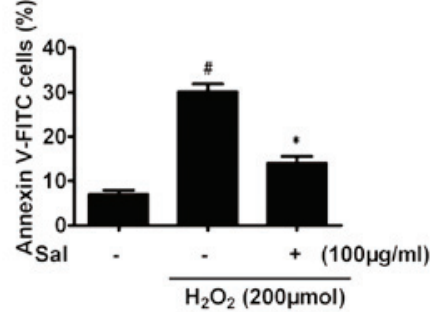

Figure 3. Sal inhibited HUVEC apoptosis induced by $\mathrm{H}_{2} \mathrm{O}_{2}$ stimulation. (A-C) Representative graphs of cells with various treatments are displayed. Number of annexin $\mathrm{V}^{+} / \mathrm{PI}^{-}$-stained cells decreased significantly in the $\mathrm{Sa}$ treatment groups. (D) Sal treatment attenuated cell death and late apoptosis in HUVECs induced by $\mathrm{H}_{2} \mathrm{O}_{2} .{ }^{*} \mathrm{P}<0.01$, compared with the control group. " $\mathrm{P}<0.01$, compared with the $\mathrm{H}_{2} \mathrm{O}_{2}$ group. HUVECs, human umbilical vein endothelial cells; Sal, salidroside.

salidroside protection against $\mathrm{H}_{2} \mathrm{O}_{2}$-induced ROS. Therefore, we investigated the levels of REDD1 and consequently, the expression of HIF- $1 \alpha$ in response to $\mathrm{H}_{2} \mathrm{O}_{2}$ and salidroside. HUVECs were stimulated with salidroside for $24 \mathrm{~h}$ and subsequently with $\mathrm{H}_{2} \mathrm{O}_{2}$ for a further $6 \mathrm{~h}$; following this, the REDD1 and HIF-1 $\alpha$ protein levels were analyzed. Incubation of HUVECs in $\mathrm{H}_{2} \mathrm{O}_{2}$ stimulated REDD1 expression (Fig. 4A). Notably, this expression was enhanced when the HUVECs were incubated with salidroside under $\mathrm{H}_{2} \mathrm{O}_{2}$. This result indicates that REDD1 may be activated under $\mathrm{H}_{2} \mathrm{O}_{2}$ conditions and salidroside. Similarly, $\mathrm{H}_{2} \mathrm{O}_{2}$ and salidroside conditions led to an increase in the levels of HIF-1 $\alpha$ protein, consistent with previous findings.
A

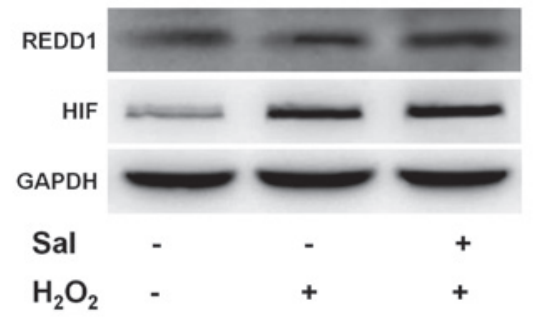

B

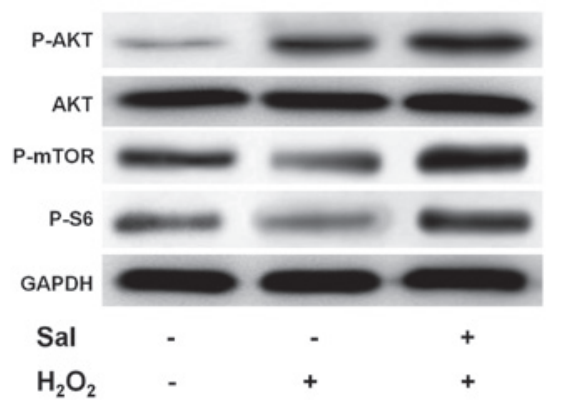

Figure 4. Sal increased the expression of REDD1 protein and activated the Akt/mTOR pathway. (A) Sal increased the expression of REDD1 protein in HUVECs under $\mathrm{H}_{2} \mathrm{O}_{2}$ stimulation. (B) Sal activated the AKT/mTOR pathway in HUVECs under $\mathrm{H}_{2} \mathrm{O}_{2}$ stimulation. Total Akt and GAPDH expression are shown as sample loading controls. The immunoblot is representative of $n=3$ experiments. REDD1, regulated in development and DNA damage responses-1; HUVECs, human umbilical vein endothelial cells; GAPDH, glyceraldehyde 3-phosphate dehydrogenase; Sal, salidroside.

In order to fully elucidate the potential signaling pathway involved in anti-apoptotic induction by salidroside under oxidative stress conditions in HUVECs, we examined the implication of protein kinases activated in response to salidroside. The mTOR pathway has been reported to enhance translation and transcription, enabling cell growth. Therefore, we investigated whether salidroside activated $\mathrm{mTOR}$ in the HUVECs treated with $\mathrm{H}_{2} \mathrm{O}_{2}$, and if so, whether Akt modulates this activation. Therefore, HUVECs were pretreated with salidroside and subjected to $6 \mathrm{~h}$ of treatment with $\mathrm{H}_{2} \mathrm{O}_{2}$. We performed western blot analysis in order to detect the effect of salidroside on the phosphorylation of Akt and mTOR in HUVECs. We observed that $\mathrm{H}_{2} \mathrm{O}_{2}$ and salidroside pretreatment increased the expression of phospho-Akt (serine 473; Fig. 4B). However, mTOR and S6 phosphorylation decreased significantly in HUVECs treated with $\mathrm{H}_{2} \mathrm{O}_{2}$ alone. Notably, phosphorylation levels were significantly elevated following pretreatment with salidroside (Fig. 4B).

Antioxidative effect of salidroside is dependent on REDDI activation. To determine whether salidroside-induced antioxidatives require the function of REDD1, we examined the effect of transfecting HUVECs with siRNAs targeting REDD1. Initially, we examined the effect of siRNAs targeting REDD1. Western blot analysis revealed that siRNAs were highly effective at decreasing REDD1 protein levels (Fig. 5A). Furthermore, REDD1 siRNAs significantly reduced the salidroside-induced antioxidative effect (Fig. 5B). Thus, salidroside protects against oxidative stress-induced cell death and this protective effect is dependent upon REDD1 activation. 
A

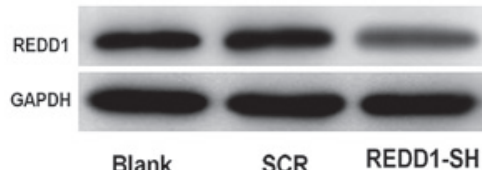

B

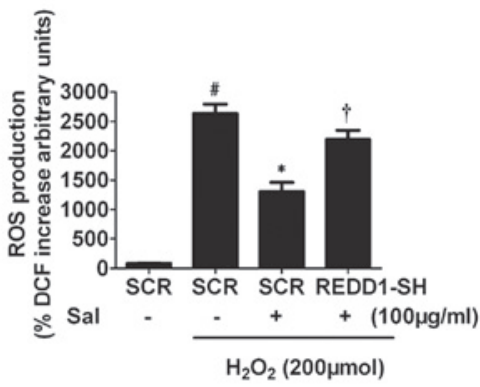

C

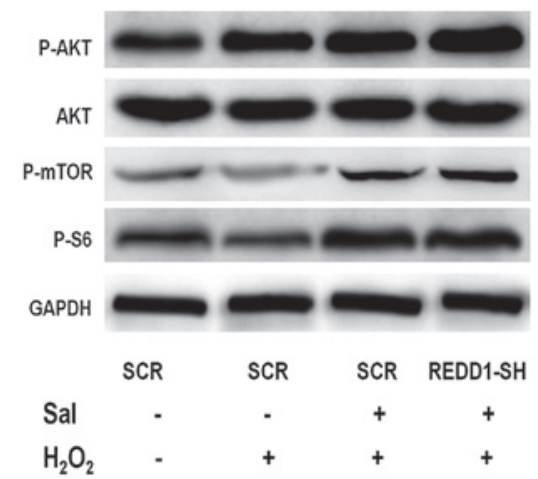

Figure 5. The antioxidative effect of Sal is dependent on REDD1 activation. (A) Knockdown of REDD1 in HUVECs by lentiviral REDD1-directed shRNA (shREDD1) or the control group, assessed by western blot analysis. GAPDH served as a loading control. (B) REDD1 knockdown decreases the antioxidative effect of Sal under $\mathrm{H}_{2} \mathrm{O}_{2}$. Equal numbers of the indicated REDD1 $\%$ HUVECs were treated under $\mathrm{H}_{2} \mathrm{O}_{2}$ with or without Sal, and increased ROS production in whole REDD1 $\%$ versus wild-type cells was measured using DCFH. ${ }^{\#} \mathrm{P}<0.01$, compared with the control. ${ }^{*} \mathrm{P}<0.01$, compared with the $\mathrm{H}_{2} \mathrm{O}_{2}$ treatment group. ${ }^{\mathrm{P}}<0.01$, compared with the SCR group. (C) Western blot analysis shows that Akt/mTOR pathway expression remained upregulated in REDD1-depleted HUVECs as well as untransfected and control siRNA HUVECs subjected to hypoxic stress. REDD1, regulated in development and DNA damage responses-1; HUVECs, human umbilical vein endothelial cells; ROS, reactive oxygen species; GAPDH, glyceraldehyde 3-phosphate dehydrogenase, DCFH, dichlorofluorescein; Sal, salidroside.

These data also confirm that activation of REDD1 is protective against oxidative stress.

PI3K participates in the regulation of $m T O R$ expression by salidroside. To determine whether PI3K activation is involved in the induction of mTOR expression by salidroside, we treated HUVECs with the PI3K inhibitor LY294002. We subjected HUVECs to salidroside for $24 \mathrm{~h}$ under $\mathrm{H}_{2} \mathrm{O}_{2}$ conditions in the absence or presence of inhibitors, and mTOR expression was detected by immunoblotting. Salidroside and $\mathrm{H}_{2} \mathrm{O}_{2}$ stimulate Akt expression, and the combination of the treatments led to an additive effect. However, LY294002 blocked the effect of salidroside on the expression of mTOR. Furthermore, LY294002 did not prevent the effect of ROS production (Fig. 6). These results suggested that in HUVECs, salidroside stimulates mTOR expression through PI3K/mTOR signaling pathways. These data demonstrate that Akt may be a central regulator of the mTOR pathway for the anti-apoptotic effect of salidroside.
A

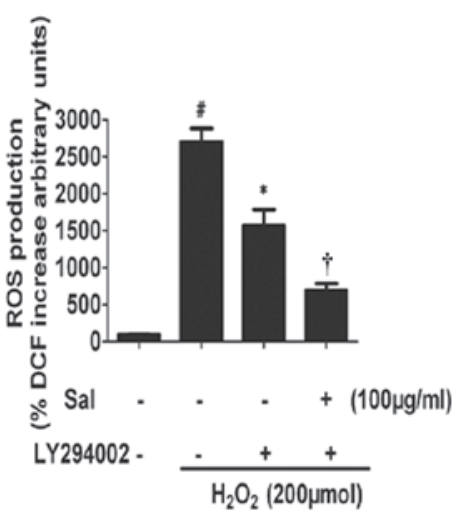

B

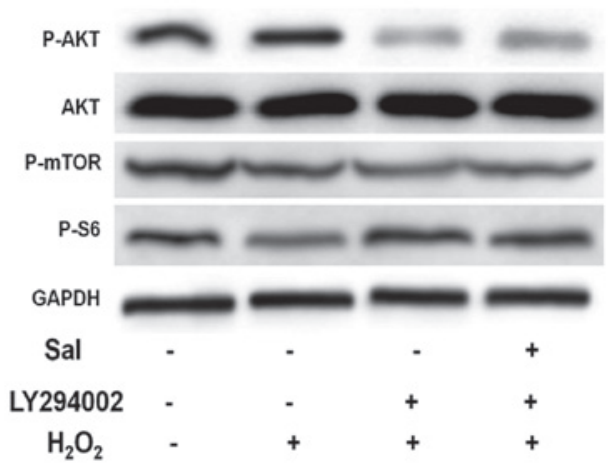

Figure 6. The PI3K pathway participates in the regulation of mTOR expression by Sal. (A) Changes in ROS production induced by either Sal or $\mathrm{H}_{2} \mathrm{O}_{2}$ treatment alone or in combination in the absence and presence of LY294002, respectively. HUVECs were preincubated with Sal, and then coincubated with $\mathrm{H}_{2} \mathrm{O}_{2}$ for a further $6 \mathrm{~h}$, and LY294002 was added 30 min prior to treatment with Sal. ${ }^{\#} \mathrm{P}<0.01$, compared with the control. ${ }^{*} \mathrm{P}<0.01$, compared with the $\mathrm{H}_{2} \mathrm{O}_{2}$ treatment group. ${ }^{\dagger} \mathrm{P}<0.01$, compared with the $\mathrm{H}_{2} \mathrm{O}_{2}+\mathrm{LY} 294002$ treatment group. (B) Activation of the mTOR pathway by $\mathrm{Sal}$ is dependent on PI3K/Akt. PI3K/Akt acted in parallel to activate the mTOR pathway following treatment with Sal. The PI3K inhibitor LY294002 blocks the increase in mTOR and PS6 protein levels induced by Sal under $\mathrm{H}_{2} \mathrm{O}_{2}$ conditions. Levels of phosphorylated Akt (Ser473), mTOR and p-p70S6K were determined by immunoblotting. Total Akt and GAPDH expression are shown as sample loading controls. A representative experiment of three independent experiments is shown. HUVECs, human umbilical vein endothelial cells; ROS, reactive oxygen species; GAPDH, glyceraldehyde 3-phosphate dehydrogenase; Sal, salidroside.

\section{Discussion}

In the present study, we demonstrated that increased REDD1 expression in response to salidroside may participate in the mechanism by which salidroside inhibited $\mathrm{H}_{2} \mathrm{O}_{2}$-induced HUVEC oxidative stress, and salidroside-induced mTOR expression occurs through the $\mathrm{PI} 3 \mathrm{~K} / \mathrm{mTOR}$ signaling pathway to protect HUVECs against apoptosis induced by $\mathrm{H}_{2} \mathrm{O}_{2}$.

The necrotic and apoptotic processes induced by oxidative stress are involved in the pathogenesis of cardiac ischemic-reperfusion injury, which may ultimately lead to heart failure $(1,18)$. Our present study confirmed that $\mathrm{H}_{2} \mathrm{O}_{2}$ significantly decreases cell viability, as evidenced by CCK-8, whereas pretreatment with various concentrations (10,50 and $100 \mu \mathrm{g} / \mathrm{ml}$ ) of salidroside attenuated the $\mathrm{H}_{2} \mathrm{O}_{2}$-decreased cell viability in a dose-dependent manner. These results indicated that salidroside exhibited an apparent protective effect against 
$\mathrm{H}_{2} \mathrm{O}_{2}$-induced cytotoxicity and inhibited ROS production in response to $\mathrm{H}_{2} \mathrm{O}_{2}$ in HUVECs.

Recently, there has been increased interest in the role of the mTOR pathway in the heart. While the evidence is mounting that the activation of mTOR may be an important cellular signaling event for preventing apoptotic processes, the role of mTOR in regulating cardioprotection is less clear in that it has been examined in a number of settings with what appear to be disparate conclusions $(19,20)$. Recent studies have examined the role of Akt in the protective effect of salidroside, and the conclusions reached were that salidroside prevented apoptosis in neuronal and cardiac cells, through the activation of the PI3K/Akt pathway $(21,22)$. These findings indicated the regulation of Akt by salidroside, which aroused our interest in the role of mTOR and the downstream target, p70S6K, in the heart. In addition, examination of the regulation of salidroside may be crucial in identifying the molecular mechanisms that regulate salidroside in the heart under conditions of oxidative stress. Thus, we aimed to further characterize the regulation of mTOR by salidroside.

Therefore, we determined whether salidroside affected the phosphorylation of the mTOR pathway in HUVECs. In this study, we identified one key anti-apoptotic role for salidroside under $\mathrm{H}_{2} \mathrm{O}_{2}$ : The activation of mTOR. The activation of mTOR pathway members was analyzed by western blot analysis using the specific antibody against the phosphorylated form of mTOR and SP6. We observed that treatment of HUVECs with salidroside increased the level of mTOR phosphorylation and SP6.

Previous studies have reported that salidroside induced the anti-apoptotic effect, so we also examined the cell apoptosis of HUVECs exposed to salidroside. As shown in Fig. 3, following the treatment of the cells with an increased concentration of salidroside for $24 \mathrm{~h}$, a significant decrease in apoptosis occurred in HUVECs, which may be correlated to the salidroside-induced upregulation of mTOR.

We demonstrated that oxidative stress inactivates mTOR. Numerous studies have demonstrated that this inactivation is dependent on HIF activity and HIF-inducible REDD1 $(12,13,23,24)$. REDD1, an important HIF-1 effector, is important in the TSC1 (hamartin)/TSC2 (tuberin)-mediated inhibition of mTOR (13). However, Hernández et al discovered that REDD-1 is downregulated by ROS by inactivating TSC2 and activating mTOR (25). However, our results indicate that stimulation with $\mathrm{H}_{2} \mathrm{O}_{2}$ increased REDD1 expression, which coincided with the upregulated expression of HIF-1 $\alpha$ in HUVECs. Furthermore, we observed that pretreatment with salidroside followed by coincubation with $\mathrm{H}_{2} \mathrm{O}_{2}$, increased REDD1 and HIF-1 $\alpha$ levels compared with those treated by $\mathrm{H}_{2} \mathrm{O}_{2}$ alone. The synergy between salidroside and $\mathrm{H}_{2} \mathrm{O}_{2}$ is due to the fact that they regulate REDD1 through distinct mechanisms. Although our data support a regulatory role for REDD1 under oxidative stress conditions, we observed the upregulation of mTOR following treatment with salidroside, which varies from the mTOR inhibition of oxidative stress occurring independently of REDD1. We propose that mTOR regulation involves multiple pathways, including mediation by the AMPK/TSC2/Rheb pathway and inhibition by the HIF/REDD1/2 pathway or the other HIF-independent pathways affected by mTOR, including PI3K/Akt. In this regard, the elucidation of the basic mechanisms affecting this network of signaling molecules will offer essential information to improve our understanding of salidroside as well as supporting the pharmacological mechanism-based application of salidroside.

Akt is an important survival kinase in cell proliferation and survival, which is activated downstream of PI3K (26). Akt-mediated protection inhibits cardiomyocyte necrotic and apoptotic death induced by deleterious stimuli (27). The existence of several possible routes by which Akt modulates the mTOR pathway suggests that this may be a central mechanism of mTOR regulation. This study aimed to identify whether PI3K signaling pathways regulated mTOR activity in HUVECs exposed to salidroside under oxidative stress conditions. Herein, we delineate a novel signaling pathway regulated by salidroside-activated Akt that leads to the activation of mTOR. This conclusion is supported by the following results: i) Under oxidative stress conditions, salidroside induces markedly higher phosphorylation levels of Akt compared with those treated by $\mathrm{H}_{2} \mathrm{O}_{2}$ alone, which is similar to the result reported by Zhu et al (22); ii) LY294002 efficiently suppressed the activation of Akt and the induction of mTOR expression by salidroside under oxidative stress conditions; iii) LY294002 inhibited the phosphorylation of mTOR; iv) the downregulation of mTOR inhibited the activation of SP6; and v) mTOR expression was unaffected by siRNAs targeting REDD1. We delineated the pathway upstream of mTOR activation in response to salidroside under oxidative stress, defining Akt as an essential activator of the mTOR pathway in this situation.

Due to the fact that REDD1 is essential in limiting the generation of ROS by an unidentified mechanism and in ROS attenuation by salidroside, we hypothesized that the mechanism by which salidroside inhibits $\mathrm{H}_{2} \mathrm{O}_{2}$-induced oxidative stress in HUVECs lies in the upregulation of the REDD1 protein. To more clearly define the role of REDD1, we examined the production of ROS in REDD1-knockdown HUVECs. The REDD1 $\%$ cells demonstrated a substantial elevation in ROS production, and treatment with salidroside was insufficient to normalize the ROS levels to wild-type levels (Fig. 5).

In conclusion, we demonstrated that salidroside protects HUVECs against oxidative injury induced by $\mathrm{H}_{2} \mathrm{O}_{2}$ and that the potential mechanisms may involve increasing REDD1 expression to prevent the generation of ROS, modulating the expression of HIF-1 $\alpha$ and regulating the activation of the PI3K/Akt pathway followed by activating the downstream molecules of mTOR and SP6 to reduce $\mathrm{H}_{2} \mathrm{O}_{2}$-induced apoptosis. These combined effects may explain the marked protective effect of salidroside.

\section{Acknowledgements}

This study was financially supported by the funding of the Natural Science Foundation of China (no. 81001561).

\section{References}

1. Hirooka Y, Sagara Y, Kishi T and Sunagawa K: Oxidative stress and central cardiovascular regulation. Pathogenesis of hypertension and therapeutic aspects. Circ J 74: 827-835, 2010.

2. Stocker R and Keaney JF Jr: Role of oxidative modifications in atherosclerosis. Physiol Rev 84: 1381-1478, 2004.

3. Suzuki YJ, Jain V, Park AM and Day RM: Oxidative stress and oxidant signaling in obstructive sleep apnea and associated cardiovascular diseases. Free Radic Biol and Med 40: 1683-1692, 2006. 
4. Kumar D and Jugdutt BI: Apoptosis and oxidants in the heart. J Lab Clin Med 142: 288-297, 2003.

5. Zhu YZ, Huang SH, Tan BK, Sun J, Whiteman M and Zhu YC: Antioxidants in Chinese herbal medicines: a biochemical perspective. Nat Prod Rep 21: 478-489, 2004.

6. Fingar DC and Blenis J: Target of rapamycin (TOR): an integrator of nutrient and growth factor signals and coordinator of cell growth and cell cycle progression. Oncogene 23: 3151-3171, 2004.

7. Proud CG: The multifaceted role of mTOR in cellular stress responses. DNA Repair (Amst) 3: 927-934, 2004.

8. Hornberger TA, Stuppard R, Conley KE, et al: Mechanical stimuli regulate rapamycin-sensitive signalling by a phosphoinositide 3-kinase-, protein kinase B- and growth factor-independent mechanism. Biochem J 380: 795-804, 2004.

9. Reiling JH and Sabatini DM: Stress and mTORture signaling. Oncogene 25: 6373-6383, 2006.

10. Peterson TR, Laplante M, Thoreen CC, et al: DEPTOR is an mTOR inhibitor frequently overexpressed in multiple myeloma cells and required for their survival. Cell 137: 873-886, 2009

11. Vander Haar E, Lee S, Bandhakavi S, Griffin TJ and Kim DH: Insulin signalling to mTOR mediated by the Akt/PKB substrate PRAS40. Nat Cell Biol 9: 316-323, 2007.

12. Sofer A, Lei K, Johannessen CM and Ellisen LW: Regulation of mTOR and cell growth in response to energy stress by REDD1. Mol Cell Biol 25: 5834-5845, 2005.

13. Brugarolas J, Lei K, Hurley RL, et al: Regulation of mTOR function in response to hypoxia by REDD1 and the TSC1/TSC2 tumor suppressor complex. Genes Dev 18: 2893-2904, 2004.

14. Shoshani T, Faerman A, Mett I, et al: Identification of a novel hypoxia-inducible factor 1-responsive gene, RTP801, involved in apoptosis. Mol Cell Biol 22: 2283-2293, 2002.

15. Ellisen LW, Ramsayer KD, Johannessen CM, et al: REDD1, a developmentally regulated transcriptional target of p63 and p53, links p63 to regulation of reactive oxygen species. Mol Cell 10: 995-1005, 2002.

16. Yu S, Liu M, Gu X and Ding F: Neuroprotective effects of salidroside in the PC12 cell model exposed to hypoglycemia and serum limitation. Cell Mol Neurobiol 28: 1067-1078, 2008.

17. Zhang J, Liu A, Hou R, et al: Salidroside protects cardiomyocyte against hypoxia-induced death: a HIF-1 alpha-activated and VEGF-mediated pathway. Eur J Pharmacol 607: 6-14, 2009.
18. Nakayama $\mathrm{H}$, Chen $\mathrm{X}$, Baines $\mathrm{CP}$, et al: $\mathrm{Ca}^{2+}{ }_{-}$and mitochondrial-dependent cardiomyocyte necrosis as a primary mediator of heart failure. J Clin Invest 117: 2431-2444, 2007.

19. Buss SJ, Muenz S, Riffel JH, et al: Beneficial effects of mammalian target of rapamycin inhibition on left ventricular remodeling after myocardial infarction. J Am Coll Cardiol 54: 2435-2446, 2009.

20. Lajoie C, El-Helou V, Proulx C, Clément R, Gosselin H and Calderone A: Infarct size is increased in female post-MI rats treated with rapamycin. Can J Physiol Pharmacol 87: 460-470, 2009.

21. Zhang L, Ding W, Sun H, et al: Salidroside protects PC12 cells from $\mathrm{MPP}^{+}$-induced apoptosis via activation of the PI3K/Akt pathway. Food Chem Toxicol 50: 2591-2597, 2012.

22. Zhu Y, Shi YP, Wu D, et al: Salidroside protects against hydrogen peroxide-induced injury in cardiac $\mathrm{H} 9 \mathrm{c} 2$ cells via PI3K-Akt dependent pathway. DNA Cell Biol 30: 809-819, 2011.

23. DeYoung MP, Horak P, Sofer A, Sgroi D and Ellisen LW: Hypoxia regulates TSC1/2-mTOR signaling and tumor suppression through REDD1-mediated 14-3-3 shuttling. Genes Dev 22: 239-251, 2008

24. Liu L, Cash TP, Jones RG, Keith B, Thompson CB and Simon MC: Hypoxia-induced energy stress regulates mRNA translation and cell growth. Mol Cell 21: 521-531, 2006.

25. Hernández G, Lal H, Fidalgo M, et al: A novel cardioprotective p38-MAPK/mTOR pathway. Exp Cell Res 317: 2938-2949, 2011.

26. Wang BH, Shravah J, Luo HL, Raedschelders K, Chen DD and Ansley DM: Propofol protects against hydrogen peroxide-induced injury in cardiac $\mathrm{H} 9 \mathrm{c} 2$ cells via Akt activation and Bcl-2 up-regulation. Biochem Biophys Res Commun 389: $105-111,2009$

27. Miyamoto S, Murphy AN and Brown JH: Akt mediated mitochondrial protection in the heart: metabolic and survival pathways to the rescue. J Bioenerg Biomembr 41: 169-180, 2009. 